\title{
Dynamic Response of Functionally Graded Circular Plate under Thermal Shock
}

\author{
Jing-Hua ZHANG ${ }^{1, a}$, Shuang-Chao PAN ${ }^{1, b, *}$ \\ ${ }^{1}$ School of Sciences, Lanzhou University of Technology, Lanzhou 730050, China \\ azjhhrb@163.com, bshch_pan@sina.com \\ ${ }^{*}$ Corresponding author
}

Keywords: Functionally Graded Materials, Circular Plate, Thermal Shock, Dynamic Response.

\begin{abstract}
Dynamic response of a functionally graded material circular plate subjected to thermal shock is investigated based on the von Kármán's plate theory. The geometric imperfections of the plate are taken into account and the bottom surface of the circular plate is subjected to uniform thermal shock loadings. The deflections of dynamic response are obtained by numerically solving the governing equations using series expansions and Runge-Kutta method. The effects of the material constitution and initial geometric imperfection of the plate on the dynamic response are discussed.
\end{abstract}

\section{Introduction}

Comprehensive works on the transient response of structures under thermal shock have been reported in the literature. Most of these researches are involved the conventional composite materials or homogeneous materials. Huang and Duan[1] studied the dynamic buckling of a circular copper plate under laser irradiation. Based on the fully coupled thermoelastic theory, thermal dynamic stability of symmetrically laminated orthotropic rectangular plates subjected to an oscillating thermal load was analyzed by Markus, et al. [2].

Functionally graded materials (FGM) have been regarded as one of the advanced inhomogeneous composite materials, usually made from metal and ceramic, taking advantage of the merits of constituent materials adequately. However, there have been few researches involving dynamic stability of FGM structures under thermal shock. Based on the classical shell theory with Sanders' nonlinear kinematic relations, a dynamic thermal post-buckling behavior of functionally graded cylindrical shells subjected to the combined action of thermal load and applied actuator voltage was analyzed by Mirzavand, et al. [3]. A finite difference based method combined with the Runge-Kutta method was employed to predict the post-buckling equilibrium paths. On the basis of the third-order shear deformation shell theory, Mirzavand, et al. [4] obtained the piezoelectric functionally graded cylindrical shell buckling equilibrium paths and dynamic buckling temperature. Mehrian and Naei[5] studied dynamic response of functionally graded partial annular disk under radial thermal shock by using a hybrid Fourier-Laplace transform in conjunction with finite element approach.

In the present paper, the dynamic response of imperfect circular FGM plates under thermal shock is investigated. Some regular conclusions are achieved through analyzing and discussing the numerical solutions in detail.

\section{Problem Formulation}

\section{Material Properties of the FGM Plate}

We consider a circular functionally graded plate with uniform thickness $h$, radius $R$. The mid-plane of the plate is referred to cylindrical coordinates $r$ and $\theta$ in the radial and the circumferential directions. Thickness direction coordinate is $z$, positive upward. The material properties of the FGM plate are assumed to vary only in the thickness direction from full metal at the top surface to full ceramic at the bottom. The volume fractions and material properties are 


$$
V_{\mathrm{c}}(z)=\left(\frac{h-2 z}{2 h}\right)^{n}, V_{m}(z)=1-V_{c}, P(z)=\left(P_{c}-P_{m}\right) V_{m}(z)+P_{m} .
$$

Where $P_{c}$ and $P_{m}$ are material properties of ceramic and metal, respectively. $V_{c}(z)$ is the volume content of the ceramic, $k$ is the volume fraction index of the ceramic.

\section{Fundamental Equations}

The corresponding displacements in the mid-surface of the circular plate are designated $u(r, t)$ and $w(r, t)$ in directions of $r$ and $z$, respectively. Considering axisymmetric deformation, the displacement $v$ in the direction of $\theta$ is zero. Linear geometrical equations and constitutive equations are expressed as

$$
\begin{aligned}
& \left\{\varepsilon_{r}, \varepsilon_{\theta}\right\}^{\mathrm{T}}=\left\{\varepsilon_{r}^{0}, \varepsilon_{\theta}^{0}\right\}^{\mathrm{T}}+z\left\{\kappa_{r}, \kappa_{\theta}\right\}^{\mathrm{T}}, \\
& \left\{\varepsilon_{r}^{0}, \varepsilon_{\theta}^{0}\right\}^{\mathrm{T}}=\left\{\frac{\partial u}{\partial r}+\frac{\partial w}{\partial r} \frac{\partial w_{0}}{\partial r}+\frac{1}{2}\left(\frac{\partial w}{\partial r}\right)^{2}, \frac{u}{r}\right\}^{\mathrm{T}},\left\{\kappa_{r}, \kappa_{\theta}\right\}^{\mathrm{T}}=\left\{-\frac{\partial^{2} w}{\partial r^{2}},-\frac{1}{r} \frac{\partial w}{\partial r}\right\}^{\mathrm{T}}, \\
& \sigma_{r}=\frac{E(z)\left(\varepsilon_{r}+v \varepsilon_{\theta}\right)}{1-v^{2}}-\frac{E(z) \alpha(z) T(z, t)}{1-v}, \sigma_{\theta}=\frac{E(z)\left(\varepsilon_{\theta}+v \varepsilon_{r}\right)}{1-v^{2}}-\frac{E(z) \alpha(z) T(z, t)}{1-v}
\end{aligned}
$$

Where $\varepsilon_{r}$ and $\varepsilon_{\theta}$ are the normal strains at an arbitrary point in the mid-surface of the deformed plate, $\kappa_{r}$ and $\kappa_{\theta}$ are curvatures, produced by the deformation, $w_{0}(r)$ is the initial geometric imperfection, $t$ is the time, $\sigma_{r}$ and $\sigma_{\theta}$ are the normal stresses in $r$ and $\theta$ directions, respectively. $T(z, t)$ is the temperature rise.

Substituting Eqs.(2a, b) into Eqs.(3a, b), integrating $\sigma_{r}$ and $\sigma_{\theta}$ along the thickness direction, the membrane forces and the bending moments per unit area are given by

$$
\begin{aligned}
& N_{r}=A\left(\varepsilon_{r}^{0}+v \varepsilon_{\theta}^{0}\right)+B\left(\kappa_{r}+v \kappa_{\theta}\right)-N^{T}, N_{\theta}=A\left(\varepsilon_{\theta}^{0}+v \varepsilon_{r}^{0}\right)+B\left(\kappa_{\theta}+v \kappa_{r}\right)-N^{T} \\
& M_{r}=B\left(\varepsilon_{r}^{0}+v \varepsilon_{\theta}^{0}\right)+D\left(\kappa_{r}+v \kappa_{\theta}\right)-M^{T}, M_{\theta}=B\left(\varepsilon_{\theta}^{0}+v \varepsilon_{r}^{0}\right)+D\left(\kappa_{\theta}+v \kappa_{r}\right)-M^{T} . \\
& \text { where }(A, B, D)=\frac{1}{1-v^{2}} \int_{-h / 2}^{h / 2} E(z)\left(1, z, z^{2}\right) \mathrm{d} z,\left(N^{T}, M^{T}\right)=\frac{1}{1-v} \int_{-h / 2}^{h / 2} E(z) \alpha(z) T(z, t)(1, z) \mathrm{d} z .
\end{aligned}
$$

\section{The Transient Temperature Field}

The dynamic response of FGM circular plate under the initial steady-state heat balance environment and suddenly subjected to uniform thermal loads on its lower surface is investigated. The temperature field of the plate varies with time and the location of the thickness direction, and its upper surface exchanges heat with the external environment. Thus, the heat conduction equation in the absence of internal heat sources reduces to

$$
C(z) \rho(z) \frac{\partial T}{\partial t}=\frac{\partial}{\partial z}\left[K(z) \frac{\partial T}{\partial z}\right],\left(t>0,-\frac{h}{2}<z<\frac{h}{2}\right) .
$$

The thermal initial conditions and the boundary conditions on the lower and the upper surfaces, are specified as

$$
T(z, 0)=0, T\left(-\frac{h}{2}, t\right)=(\Delta T)\left(1-e^{-a t}\right),-\left.K(z) \frac{\partial T}{\partial z}\right|_{z=h / 2}=h_{r} T\left(\frac{h}{2}, t\right) .
$$

Where $h_{r}$ is the heat exchange coefficient between the upper surface of the plate and the environment. 
The Laplace transformation technique and the power series method are employed to solve Eps $(5,6)$ so the temperature can be obtained.

\section{Motion Equations and Dynamic Governing Equations}

Equilibrium equations of the circular plate with axisymmetric deformations are derived in terms of the resultant forces as follows:

$$
\frac{\partial\left(r N_{r}\right)}{\partial r}-N_{\theta}=0, \frac{\partial^{2}\left(r M_{r}\right)}{\partial r^{2}}-\frac{\partial M_{\theta}}{\partial r}+\frac{\partial}{\partial r}\left(r N_{r}\left(\frac{\partial w}{\partial r}\right)\right)-I_{0} r \frac{\partial^{2} w}{\partial t^{2}}=0
$$

Where $I_{0}=\int_{-h / 2}^{h / 2} \rho(z) \mathrm{d} z$ is the mass per unit area.

Substitute Eqs. (2a, b), (4a, b) into Eqs. (7a, b) and introduce the following dimensionless quantities for an easy solution.

$$
\begin{aligned}
& x=\frac{r}{R},\left(W, W_{0}\right)=\frac{\left(w, w_{0}\right)}{h}, \tau=t \sqrt{\frac{E_{m}}{\rho_{m} h^{2}}}, U=\frac{u R}{h^{2}}, D_{1}=\frac{A}{h E_{m}}, D_{2}=\frac{B}{h^{2} E_{m}}, D_{3}=\frac{D}{h^{3} E_{m}}, D_{4}=\frac{h}{R}, \\
& N_{T}=\frac{N^{T} R^{2}}{E_{m} h^{3}} .
\end{aligned}
$$

Where $E_{m}$ is the Young's modulus of metal material. Therefore, the dimensionless governing equations are

$$
\frac{\partial}{\partial x}\left[\frac{1}{x} \frac{\partial}{\partial x}(x U)\right]=\frac{D_{2}}{D_{1}} F_{1}+F_{2}, \quad D_{3} \nabla^{4} W=-N_{T} F_{3}+D_{2}\left(F_{4}+F_{5}\right)+D_{1}\left(F_{6}+F_{7}\right)-\frac{D_{0}}{D_{4}{ }^{4}} \frac{\partial^{2} W}{\partial \tau^{2}} .
$$

in which the linear differential operators $\nabla^{4}=\frac{\partial^{4}}{\partial x^{4}}+\frac{2}{x} \frac{\partial^{3}}{\partial x^{3}}-\frac{1}{x^{2}} \frac{\partial^{2}}{\partial x^{2}}+\frac{1}{x^{3}} \frac{\partial}{\partial x}, F_{1} \sim F_{7}$ are partial differential expressions of $U$ and $W$, given in the Appendix.

The edge of the plate considered is simply supported, so the dimensionless boundary conditions at the edge and the continuous conditions at the center of the plate are written as

$$
\mathrm{x}=0: U=0, W=\delta, \frac{\partial W}{\partial x}=0, \lim _{x \rightarrow 0}\left(\frac{1}{x} \frac{\mathrm{d}^{2} W}{\mathrm{~d} x^{2}}+\frac{\mathrm{d}^{3} W}{\mathrm{~d} x^{3}}\right)=0, \mathrm{x}=1: W=0, U=0, \frac{\partial^{2} W}{\partial x^{2}}=0
$$

Assume that the circular FGM plate axisymmetric deflection of expression is a power series[1], this is

$$
W=\delta\left(1+\sum_{i=2,4}^{\infty} A_{i} x^{i}\right) \approx \delta\left(1+A_{2} x^{2}+A_{4} x^{4}\right), W_{0}=\delta_{0}\left(1+A_{2} x^{2}+A_{4} x^{4}\right) .
$$

Where simply supported edge $A_{2}=-\frac{2(3+v)}{5+v}, A_{4}=-\frac{1+v}{5+v}, \delta, \delta_{0}$ is the maximum deflection and initial deflection at the center point of plate.

Substituting Eqs.(10a, b) into Eqs.(8a, b) , and integrating the dimensionless governing equations, yields

$$
\begin{aligned}
& U=\eta_{1} \delta^{2}+\eta_{2} \delta+C_{1} x+\frac{C_{2}}{x}, \\
& D_{3} D_{4} W=\lambda_{1} \delta^{3}+\lambda_{2} \delta^{2}+\lambda_{3} \delta+\lambda_{4} \ddot{\delta}+\lambda_{5}+\frac{C_{3} x^{2}(\ln x-1)}{4}+\frac{C_{4} x^{2}}{4}+C_{5} \ln x+C_{6} .
\end{aligned}
$$

Where $C_{1} \sim C_{6}$ are constants of integration, $\eta_{1}, \eta_{2}, \lambda_{1} \sim \lambda_{5}$ polynomial for $x$. Use the boundary conditions $U=0$ at $x=0$ and $x=1$, we have 


$$
\begin{aligned}
C_{1}= & {\left[\frac{(7-v) A_{4}^{2}}{6}+\frac{(5-v) A_{2} A_{4}}{3}+\frac{(3-v) A_{2}^{2}}{4}\right] \delta^{2}+\left(\frac{(3-v) A_{2}^{2}}{2}+\frac{(7-v) A_{4}^{2}}{3}+\frac{(10-2 v) A_{2} A_{4}}{3}\right) \delta_{0} \delta } \\
& -\frac{4 D_{2} A_{4}}{D_{1}} \delta, \\
C_{2}= & 0 .
\end{aligned}
$$

Substituting Eq.(11a) into Eq.(11b), in view of the finiteness of $W=\delta$ and $\lim _{x \rightarrow 0}\left(\frac{1}{x} \frac{\mathrm{d}^{2} W}{\mathrm{~d} x^{2}}+\frac{\mathrm{d}^{3} W}{\mathrm{~d} x^{3}}\right)=0$ at $x=0$, we have $C_{3}=0, C_{5}=0$.

Use the boundary conditions at $x=1$ will be $W=0, \frac{\partial^{2} W}{\partial x^{2}}=0$, we obtained

$C_{4}=B_{1} \delta^{3}+B_{2} \delta^{2}+B_{3} \delta+B_{4}+B_{5} \ddot{\delta}, C_{6}=B_{6} \delta^{3}+B_{7} \delta^{2}+B_{8} \delta+B_{9}+B_{10} \ddot{\delta}$.

Detailed expressions of polynomial $B_{1} \sim B_{10}$ are given in the Appendix. The Eqs.(11b) will be solved numerically by using Runge-Kutta method.

\section{Numerical Results and Discussions}

In the computation, a circular FGM plate made of the constituents of $\mathrm{SiC}$ and $\mathrm{Ni}$ is considered. Poisson's ratio of the plate is constant and spedified as $v=0.3$. The material properties of the constituents are reference to literature [6]. The geometries of the FGM circular plate are $R=0.2 \mathrm{~m}, h=0.01 \mathrm{~m}$. The lower surface is subjected to thermal shock loading $T(-h / 2, t)=\Delta T\left(1-e^{-a t}\right)$, here $\Delta T=300 \mathrm{~K}, a=10$. Given the heat exchange coefficient is $h_{r}=50$.

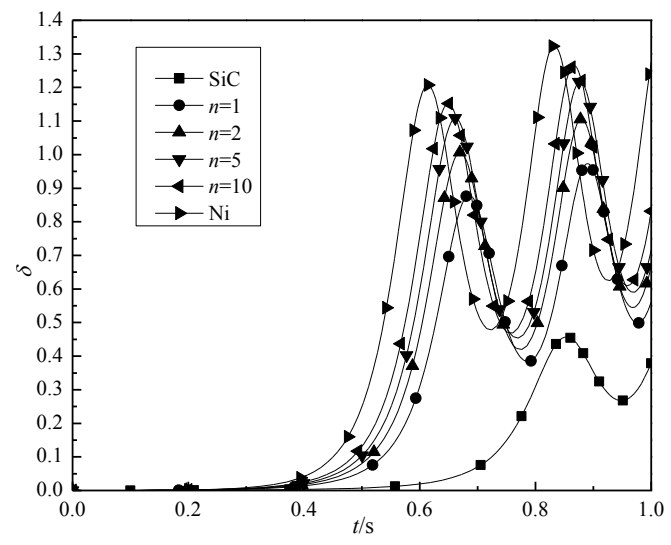

Fig. 1 The Central Deflection Responses of the FGM Circular Plate with Different $n$

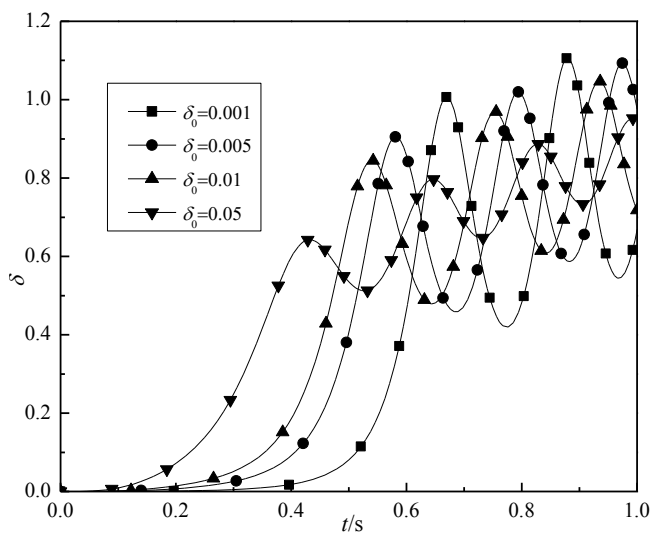

Fig. 2 The Central Deflection Responses of the FGM Circular Plate for Some Specified Imperfections

For some specified values of volume fraction index $n$ at $\delta_{0}=0.001$, Fig. 1 shows the transient central deflections in the middle surfaces of the FGM circular plate under thermal shock. The maximum central deflections of the metal and ceramic FGM circular plate are intermediate to those of the metal and the ceramic plate and increase monotonously with the increasing of volume fraction index $n$ for thermal shock. Given value of power law index $n=2$, Fig. 2 shows the dynamic response of the plate is very sensitive to the magnitude of the initial imperfect. Similar discussions can be found in literatures $[1,6]$.

\section{Summary}

Dynamic response of a functionally graded material circular plate subjected to thermal shock is investigated by using the series expansions techincal and Runge-Kutta method. It is found that the maximum central deflections increase monotonously with the increasing of volume fraction index $\mathrm{n}$ for thermal shock. The ability of the structure to withstand thermal shock is reduced with the increasing of 
power law index $n$. And the dynamic response of the plate is very sensitive to the magnitude of the initial imperfect.

\section{Acknowledgement}

This work was financially supported by the National Natural Science Foundation of China (Nos.11262010,11272278). The authors gratefully acknowledge the support.

\section{References}

[1]C. G. Huang, Z.P. Duan, Studies on the dynamic buckling of circular plate irradiated by laser beam [J], Applied Mathematics and Mechanics. 23 (2002) 667-672.

[2]S. Markus, J.B. Greenberg, Y. Stavsky, Coupled thermoelastic theory for dynamic stability of composite plates [J], Journal of Thermal Stresses. 18 (1995) 335-357.

[3]B. Mirzavand, M.R. Eslami, M. Shakeri, Dynamic thermal postbuckling analysis of piezoelectric functionally graded cylindrical shells [J], Journal of Thermal Stresses. 33 (2010) 646-660.

[4]B. Mirzavand, M.R. Eslami, J.N. Reddy, Dynamic thermal postbuckling analysis of shear deformable Piezoelectric-FGM cylindrical shells [J], Journal of Thermal Stresses. 36 (2013) 189-206.

[5]S.M.N. Mehrian, M. H. Naei, Two dimensional analysis of functionally graded partial annular disk under radial thermal shock using hybrid Fourier-Laplace transform [J], Applied Mechanics and Materials. 436 (2013) 92-99.

[6]S.R. Li, L.L. Fan, Dynamic responses of functionally graded material beams under thermal shock [J], Journal of Vibration Engineering. 22 (2009) 371-378(in Chinese).

\section{Appendix}

$$
\begin{aligned}
F_{1}= & \frac{\partial^{3} W}{\partial x^{3}}+\frac{1}{x} \frac{\partial^{2} W}{\partial x^{2}}-\frac{1}{x^{2}} \frac{\partial W}{\partial x}, F_{2}=-\left(\frac{\partial^{2} W}{\partial x^{2}}-\frac{\partial^{2} W_{0}}{\partial x^{2}}+\frac{1-v}{2 x} \frac{\partial W}{\partial x}\right) \frac{\partial W}{\partial x}-\left(\frac{\partial^{2} W}{\partial x^{2}}+\frac{1-v}{x} \frac{\partial W}{\partial x}\right) \frac{\partial W_{0}}{\partial x}, \\
F_{3}= & \frac{\partial^{2} W}{\partial x^{2}}+\frac{\partial^{2} W_{0}}{\partial x^{2}}+\frac{1}{x}\left(\frac{\partial W}{\partial x}+\frac{\partial W_{0}}{\partial x}\right), F_{4}=\frac{1}{x} \frac{\partial}{\partial x} x \frac{\partial}{\partial x} \frac{1}{x} \frac{\partial}{\partial x}(x U), \\
F_{5}= & \frac{1-3 v}{x} \frac{\partial W}{\partial x} \frac{\partial^{2} W}{\partial x^{2}}+\frac{1-2 v}{x} \frac{\partial^{2} W}{\partial x^{2}} \frac{\partial W_{0}}{\partial x}+\left[\frac{2(1-v)}{x} \frac{\partial W}{\partial x}+\frac{\partial^{2} W}{\partial x^{2}}\right] \frac{\partial^{2} W_{0}}{\partial x^{2}}+\frac{\partial W}{\partial x} \frac{\partial^{3} W_{0}}{\partial x^{3}}, \\
F_{6}= & \frac{1}{x} \frac{\partial}{\partial x}\left[x\left(\frac{\partial U}{\partial x}+\frac{v}{x} U\right) \frac{\partial W}{\partial x}\right]+\left[\frac{\partial U}{\partial x}+\frac{v}{x} U\right] \frac{\partial^{2} W_{0}}{\partial x^{2}}+\left(\frac{\partial^{2} U}{\partial x^{2}}+\frac{1+v}{x} \frac{\partial U}{\partial x}\right) \frac{\partial W_{0}}{\partial x} \\
F_{7}= & \frac{1}{x} \frac{\partial}{\partial x}\left[\frac{x}{2}\left(\frac{\partial W}{\partial x}\right)^{2} \frac{\partial W}{\partial x}\right]+\left(2 \frac{\partial W_{0}}{\partial x}+\frac{3}{2} \frac{\partial W}{\partial x}\right) \frac{\partial W}{\partial x} \frac{\partial^{2} W_{0}}{\partial x^{2}}+\left(3 \frac{\partial W}{\partial x}+\frac{\partial W_{0}}{\partial x}\right) \frac{\partial^{2} W}{\partial x^{2}} \frac{\partial W_{0}}{\partial x} \\
& +\left(\frac{3}{2 x} \frac{\partial W}{\partial x}+\frac{1}{x} \frac{\partial W_{0}}{\partial x}\right) \frac{\partial W}{\partial x} \frac{\partial W_{0}}{\partial x}, \\
B_{1}= & \left(0.208 A_{2}^{2}+0.375 A_{4}^{2}-0.953-0.375 v^{2} A_{4}^{2}-0.953 v-0.208 v^{2} A_{2}^{2}\right) D_{1} D_{4}^{4} A_{2} \\
& +\left(0.486 A_{2}^{2}-1.059 v-0.122 v^{2} A_{4}^{2}-0.486 v^{2} A_{2}^{2}-1.059+0.122 A_{4}^{2}\right) D_{1} D_{4}^{4} A_{4}
\end{aligned}
$$




$$
\begin{aligned}
& B_{2}=\left(0.367 A_{4}{ }^{2}-3.177 v+1.125 A_{2} A_{4}-3.177+1.458 A_{2}{ }^{2}-1.458 A_{2}{ }^{2} v^{2}-0.367 A_{4}{ }^{3} v^{2}\right) D_{1} D_{4}{ }^{4} A_{4} \delta_{0} \\
& +\left(0.625 A_{2}{ }^{2}-1.125 A_{4}{ }^{2} v^{2}-0.625 A_{2}{ }^{2} v^{2}\right) D_{1} D_{4}{ }^{4} A_{2} \delta_{0}+\left(1.635+1.635 v+3.333 A_{2} v\right) D_{2} D_{4}{ }^{4} A_{4} \\
& +\left(1.472+3 A_{2}-2.859 v \delta_{0}+3 A_{2} v-2.859 \delta_{0}+1.472 v+3.333 A_{4}\right) D_{2} D_{4}{ }^{4} A_{2} \\
& B_{3}=\left[0.972 A_{2} A_{4}+0.417 A_{2}{ }^{2}\left(1-v^{2}\right)+0.75 A_{4}{ }^{2}\left(1-v^{2}\right)-1.906(v+1)\right] D_{1} D_{4}{ }^{4} A_{2} \delta_{0}{ }^{2}-\frac{24 D_{2} D_{4}{ }^{4} A_{4}}{D_{1}} \\
& +\left[0.244 A_{4}{ }^{2}\left(1-v^{2}\right)-0.972 A_{2}{ }^{2} v^{2}-2.118(v+1)\right] D_{1} D_{4}{ }^{4} A_{4} \delta_{0}{ }^{2}+\left(1.5 A_{2}+1.667 A_{4}\right) D_{4}{ }^{4} N_{T} \\
& +\left[3 A_{2}(v+1)+1.477+3.333 A_{4}(v+1)+1.472 v\right] D_{2} D_{4}{ }^{4} A_{2} \delta_{0}+1.635(v+1) D_{2} D_{4}{ }^{4} A_{4} \delta_{0} \\
& B_{4}=1.667 A_{4} D_{4}^{4} \delta_{0} N_{T}+1.5 A_{2} D_{4}^{4} \delta_{0} N_{T}, B_{5}=0.375 D_{0}+0.104 D_{0} A_{2}+0.049 D_{0} A_{4} \text {, } \\
& B_{6}=\left(0.199 v+0.049 v^{2} A_{2}^{2}-0.049 A_{2}^{2}-0.092 A_{4}^{2}+0.199+0.092 v^{2} A_{4}^{2}-0.117 A_{2} A_{4}\right) D_{1} D_{4}^{4} A_{2} \\
& +\left(0.247 v+0.03 v^{2} A_{4}^{2}+0.0117 v^{2} A_{2}^{2}+0.247-0.03 A_{4}^{2}\right) D_{1} D_{4}^{4} A_{4}, \\
& B_{7}=\left[\left(0.146 A_{2}{ }^{2}+0.275 A_{4}{ }^{2}\right)\left(v^{2}-1\right)+0.596(v+1)+0.352 A_{2} A_{4} v^{2}\right] D_{1} D_{4}{ }^{4} A_{2} \delta_{0}-0.352 A_{2}{ }^{2} D_{1} D_{4}{ }^{4} A_{4} \delta_{0} \\
& +\left[0.741(v+1)+0.09 A_{4}{ }^{2}\left(v^{2}-1\right)\right] D_{1} D_{4}{ }^{4} A_{4} \delta_{0}-\left[\left(0.625 A_{2}+0.307\right)(v+1)+0.778 A_{4}\right] D_{2} D_{4}{ }^{4} A_{2} \\
& -\left[0.382(v+1)+0.778 A_{2} v\right] D_{2} D_{4}^{4} A_{4} \\
& B_{8}=\left[0.397(v+1)+0.097 A_{2}^{2}\left(v^{2}+1\right)+0.183\left(v^{2}-1\right) A_{4}{ }^{2}\right] D_{1} D_{4}{ }^{4} A_{2} \delta_{0}{ }^{2}-\left(0.313 A_{2}-0.389 A_{4}\right) D_{4}{ }^{4} N_{T} \\
& +\left(0.06 A_{4}^{2}+0.234 A_{2}^{2}\right)\left(v^{2}-1\right) D_{1} D_{4}^{4} \delta_{0}^{2} A_{4}+\left(0.494 \delta_{0}-0.382\right)(v+1) D_{1} D_{4}{ }^{4} A_{4} \delta_{0}+\frac{5 D_{2}{ }^{2} D_{4}{ }^{4} A_{4}}{D_{1}} \\
& -\left(0.307+0.625 A_{2}+0.778 A_{4}\right)(v+1) D_{2} D_{4}^{4} A_{2} \delta_{0}, \\
& B_{9}=0.389 A_{4} D_{4}^{4} \delta_{0} N_{T}+0.313 A_{2} D_{4}^{4} \delta_{0} N_{T}, B_{10}=0.078 D_{0}+0.024 D_{0} A_{2}+0.112 D_{0} A_{4} \text {. }
\end{aligned}
$$

